\title{
Effect of resveratrol on proliferation and apoptosis of human pancreatic cancer MIA PaCa-2 cells may involve inhibition of the Hedgehog signaling pathway
}

\author{
YONG QIN ${ }^{1,2^{*}}$, ZHENHUA MA $^{1}$, XIAOYAN DANG $^{3 *}$, WEI LI $^{1}$ and QINGYONG MA ${ }^{1}$ \\ ${ }^{1}$ Department of Hepatobiliary Surgery, First Affiliated Hospital of Medical College, Xi'an Jiaotong University, \\ Xi'an, Shaanxi 710061; ${ }^{2}$ Department of General Surgery, Affiliated Xi'an Central Hospital; ${ }^{3}$ Department of Emergency, \\ Second Affiliated Hospital of Medical College, Xi'an Jiaotong University, Xi'an, Shaanxi 710004, P.R. China
}

Received December 15, 2013; Accepted June 14, 2014

DOI: $10.3892 / \mathrm{mmr} .2014 .2511$

\begin{abstract}
We previously demonstrated that resveratrol (Res) regulates the expression of PKC $\alpha$ and $\delta$, to eventually inhibit growth and induce apoptosis in human gastric cancer cells. In the present study, the effect of Res on the growth of human pancreatic cancer cells was investigated, to further unveil the underlying mechanism. The human pancreatic cancer cell line MIA PaCa-2 was treated with three different concentrations of Res. Cell proliferation was assessed by the MTT assay, and apoptosis was detected by flow cytometry. Reverse transcription-quantitative PCR (RT-qPCR) and western blot analysis were used to measure the mRNA and protein levels of the Hedgehog $(\mathrm{Hh})$ signaling proteins Ihh, Ptch and Smo. Our results revealed that Res can inhibit the cell proliferative ability in a time- and dose-dependent manner. The number of formed colonies in the Res- and 5-Fu (positive control)-treated groups was decreased as compared to the negative control group. Res further induced apoptosis of MIA PaCa- 2 cells in a dose-dependent manner. In addition, the levels of Ihh, Ptch and Smo were decreased by Res treatment. Our findings suggest that Res inhibits proliferation and induces apoptosis of MIA PaCa-2 pancreatic cancer cells in vitro, which may be related to its inhibitory effect on the Hh signaling pathway.
\end{abstract}

\section{Introduction}

As an aggressive malignant disease, pancreatic cancer (PC) is the fourth leading cause of cancer mortality worldwide (1). Despite a number of in-depth studies on PC conducted in the

Correspondence to: Dr Qingyong Ma, Department of Hepatobiliary Surgery, First Affiliated Hospital of Medical College, Xi'an Jiaotong University, 277 West Yanta Road, Xi'an, Shaanxi 710061, P.R. China

E-mail: qyma56@mail.xjtu.edu.cn

*Contributed equally

Key words: resveratrol, proliferation, apoptosis, Hedgehog past few decades, the mortality rate of PC patients is nearly equal to its incidence (2). Since traditional chemotherapy and radiotherapy are ineffective on this malignancy, the 5-year survival rate of $\mathrm{PC}$ patients remains $<5 \%$, and the survival time following diagnosis is no more than one year in most patients $(1,3)$.

In recent years, research on the molecular mechanisms underlying PC has led to the development of new treatment strategies, such as the inhibition of relevant signaling pathways, gene therapy and immunotherapy. Proteins of the Hedgehog ( $\mathrm{Hh})$ signaling pathway, an important regulator of human embryonic development, are highly expressed in pancreatic cancer tissues or cells, and their expression appears to correlate to the occurrence, development and biological behavior of tumor cells (4). Hh signaling activation is a common event in pancreatic cancer (5). The Hh protein functions by binding to a 12-transmembrane domain receptor called patched 1 (Ptch1). The binding of Hh to Ptch1 allows Ptch1 to inhibit smoothened (SMO), a 7-transmembrane domain protein. The activated SMO then relocalizes to the primary cilia, and initiates an intracellular signaling cascade that eventually leads to the activation of the Gli-1 transcription factor and the upregulation of the downstream target genes, which include Ptch1 (6,7).

Resveratrol (Res) is a polyphenolic compound (trans-3,5,4'-tri-hydroxystilbene) found in grapes and 72 additional species (belonging to 12 families, 32 genera). Its highest content is reported in the fresh root of the traditional Chinese medical plant Polygonum cuspidatum (8). Previous studies have shown that resveratrol has numerous biological properties, including anti-inflammatory, antioxidant and antitumorigenic effects (9-11). Our recent study also showed that Res can suppress PC cell migration, invasion, and the progression of the epithelial-to-mesenchymal transition through the inhibition of the PI-3K/Akt/NF- $\kappa \mathrm{B}$ signaling pathway (12).

The present study investigated whether Res affects the proliferation and apoptosis of human MIA PaCa-2 PC cells, and the expression of proteins of the Hh signaling pathway. This study may provide a new therapeutic strategy for PC, as well as an experimental basis for the clinical application of Res. 


\section{Materials and methods}

Reagents. The human PC cell line MIA PaCa-2 was obtained from the Peking Union Medical College (Beijing, China). Dulbecco's modified Eagle's medium (DMEM), trypsin and fetal bovine serum (FBS) were from Hyclone ${ }^{\mathrm{TM}}$ (Thermo Fisher Scientific, Waltham, MA, USA). Res (purity >99.9\%), which was obtained from Sigma-Aldrich (St. Louis, MO, USA), was dissolved in dimethyl sulfoxide (DMSO) to obtain a $400 \mu \mathrm{mol} / 1$ stock solution. 5-Fluorouracil (5-Fu) was purchased from Xudong Haipu Pharmaceutical Co., Ltd. (Shanghai, China). 3-(4,5-Dimethylthiazol-2-yl)-2,5-diphenyltetrazolium bromide (MTT) was purchased from Sigma-Aldrich. The Annexin V-FITC apoptosis kit was purchased from Zhu hai Jian Kang yuan Co. (Zhuhai, China). The primary antibodies against Indian hedgehog (Ihh), Ptch and Smo were obtained from Santa Cruz Biotechnology, Inc. (Santa Cruz, CA, USA). The primers were designed and synthesized by Takara Bio (Dalian, China).

Cell culture and groups. The human PC cell line MIA PaCa-2 was cultured in DMEM containing 10\% dialyzed heat-inactivated FBS, $100 \mathrm{U} / \mathrm{ml}$ penicillin and $100 \mu \mathrm{g} / \mathrm{ml}$ streptomycin in a humidified atmosphere (95\% relative humidity) of $5 \% \mathrm{CO}_{2}$ at $37^{\circ} \mathrm{C}$. Cells at $70-80 \%$ confluence were digested and passaged with $2.5 \mathrm{~g} / 1$ trypsin. Cells at the logarithmic growth phase were used in all the experiments.

Cells were divided into five groups: high concentration Res $(200 \mu \mathrm{mol} / \mathrm{l})$, medium concentration Res $(100 \mu \mathrm{mol} / \mathrm{l})$, low concentration Res $(50 \mu \mathrm{mol} / \mathrm{l})$, the positive control group $(0.75 \mathrm{mg} / \mathrm{ml} \mathrm{5-Fu})$, and the negative control group $(0 \mu \mathrm{mol} / \mathrm{l}$ Res in DMEM-10\% FBS).

MTT assay. Cells were seeded in a 96-well plate at a $1 \times 10^{3}$ cells/well density, and kept at $37^{\circ} \mathrm{C}$ overnight. When the cells had reached $70 \%$ confluence, they were divided into five groups, each including 5 wells, in a final volume of $100 \mu$ l. After 24, 48 and $72 \mathrm{~h}$, respectively, $20 \mu \mathrm{l}$ of MTT solution $(5 \mathrm{~g} / \mathrm{l})$ were added to each well. Following an additional 4-h incubation, the medium was removed, and $150 \mu 1$ of DMSO were added to each well, followed by $10 \mathrm{~min}$ shaking. Finally, the optical density (OD) was measured at $490 \mathrm{~nm}$ on a spectra microplate multilabel counter reader (Victor 2-1420-015; Perkin Elmer, Waltham, MA, USA). The cell inhibition rate (\%) was calculated as $=\left(\mathrm{OD}_{\text {control }}-\mathrm{OD}_{\text {sample }}\right) /\left(\mathrm{OD}_{\text {control }}\right) \times 100$.

Colony formation assay. Cells were digested into a single cell suspension, and 200 cells were seeded in a 24-well plate. Following adherence, the cells were divided into five groups, with 4 wells in each group, and were incubated at $37^{\circ} \mathrm{C}$ in a humidified atmosphere containing $5 \% \mathrm{CO}_{2}$ for 6 days. Cell colonies were then stained with crystal violet (Sigma-Aldrich), and were manually counted under a XDS-1B microscope (Nikon, Tokyo, Japan). Only colonies containing $>50$ cells were counted. The colony formation rate $(\%)$ was calculated as $=($ median colony number/number of seeded cells) $\mathrm{x} 100$.

Annexin V-propidium iodide (PI) assay. Cells were inoculated in 25-ml flasks and were divided into five groups following adherence. The cells were harvested following exposure to
Res, 5-Fu and DMEM for $24 \mathrm{~h}$. The cells were then centrifuged at $1,500 \mathrm{x} \mathrm{g}$ for $5 \mathrm{~min}$ and collected, followed by resuspension in binding buffer consisting of $10 \mathrm{mmol} / \mathrm{l} \mathrm{HEPES}-\mathrm{NaOH}$ (pH 7.4), $140 \mathrm{mmol} / \mathrm{l} \mathrm{NaCl}$ and $2.5 \mathrm{mmol} / \mathrm{l} \mathrm{CaCl}{ }_{2}$. The samples were then incubated with $5 \mu \mathrm{l}$ Annexin $\mathrm{V}$ in the dark for $10 \mathrm{~min}$, washed with binding buffer, and resuspended in binding buffer supplemented with $1 \%$ formaldehyde, at $40^{\circ} \mathrm{C}$ for 30 min. After an additional wash in binding buffer, the cells were stained with $500 \mu \mathrm{l}$ PI for $15 \mathrm{~min}$, and were then analyzed by flow cytometry (BD Biosciences, San Jose, CA, USA). The percentage of apoptotic cells was determined using the CellQuest software (BD Biosciences). Early apoptotic cells were identified as cells that were PI-negative and Annexin V-positive.

Reverse transcription-quantitative PCR (RT-qPCR). Total RNA was extracted from the PC cells using the Fastgen200 RNA isolation system (Voerson Technology Co, Xi'an, Shaanxi, China) according to the manufacturer's protocol. Total RNA was reverse transcribed into cDNA using the RevertAid ${ }^{\mathrm{TM}}$ First Strand cDNA Synthesis kit (MBI Fermentas Inc., Burlington, ON, Canada). The primer sequences were as follows: Ihh forward (F), 5'-TCG CCC TGT GGA TGA CTG AG-3', and reverse (R), 5'-CAG AGT CTT CAG AGA CAG CCA GGA-3'; Smo F, 5'-TCA GGA TGC GTC CAC CAA GAA G-3', and R, 5'-TGT GTC CAC GGC GGC AAT C-3'; Ptch F, 5'-TGC GTG TGG AGT ATT TGG ATG AC-3', and R, 5'-CAG TGT GAT GAT GGT GAG GAT GG-3'; $\beta$-actin F, 5'-GAC TTA GTT GCG TTA CAC CCT TTC T-3', and R, 5'-GAA CGG TGA AGG TGA CAG CAG T-3'.

The PCR amplification was performed using the CFX Manager 2.1 fluorescent quantitative PCR kit (Bio-Rad Laboratories, Hercules, CA, USA), under the following conditions: $30 \mathrm{sec}$ at $95^{\circ} \mathrm{C}$, followed by 40 cycles at $95^{\circ} \mathrm{C}$ for $5 \mathrm{sec}$, at $60^{\circ} \mathrm{C}$ for $30 \mathrm{sec}$, and at $72^{\circ} \mathrm{C}$ for $30 \mathrm{sec}$. Following qPCR, a dissociation curve analysis was conducted. Relative gene expression was calculated using the $2^{-\Delta \Delta C t}$ method (13). Each measurement was performed in triplicate.

Western blot analysis. Proteins were electrophoretically resolved on a denaturing sodium dodecyl sulfate polyacrylamide gel and electrotransferred onto nitrocellulose membranes. The membranes were initially blocked with $5 \%$ nonfat dry milk in Tris-buffered saline for $2 \mathrm{~h}$, and then incubated with antibodies targeting Ihh, Ptch, Smo or $\beta$-actin. Following incubation with the primary antibodies at $4^{\circ} \mathrm{C}$ overnight, the membranes were hybridized with the horseradish peroxidase-conjugated polyclonal mouse anti-human IgG secondary antibody (1:2,000; Pierce, Rockford, IL, USA). The positive bands were visualized with an enhanced chemiluminescence system (Millipore, Billerica, MA, USA) according to the manufacturer's instructions.

Statistical analysis. Statistical analysis was performed using the SPSS software version 12.0 (SPSS Inc., Chicago, IL, USA). Differences between two groups were analyzed by two-tailed Student's t-tests, and among three or more groups by a one-way analysis of variance (ANOVA). Values were expressed as mean $\pm \mathrm{SD}$, and $\mathrm{P}<0.05$ was considered to indicate statistically significant differences. 


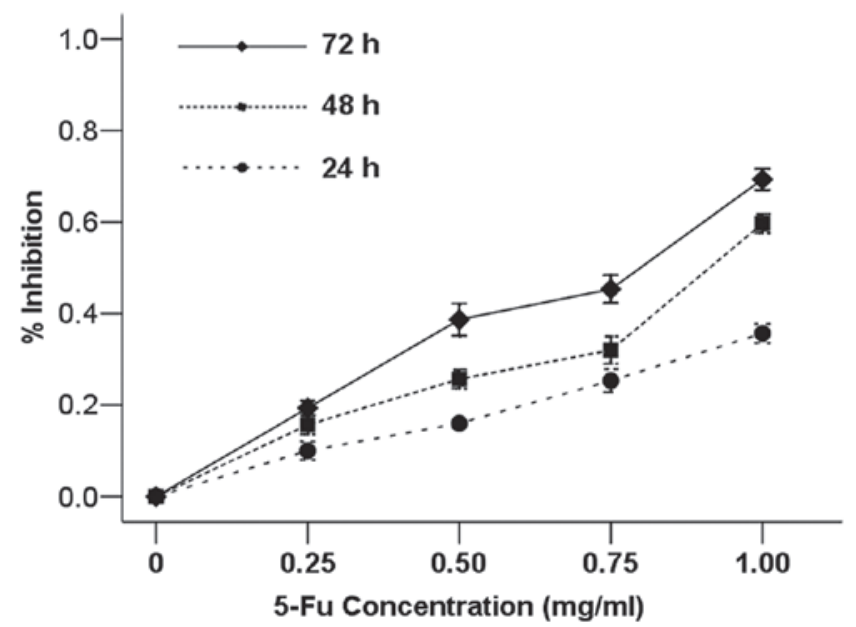

Figure 1. 5-Fluorouracil (5-Fu) inhibits the proliferation of MIA PaCa-2 pancreatic cancer (PC) cells. PC cells were treated with increasing concentrations of $5-\mathrm{Fu}(0,0.25,0.5,0.75$ and $1.00 \mathrm{mg} / \mathrm{ml})$ for 24,48 or $72 \mathrm{~h}$, and the inhibition rate of their proliferative ability was calculated.

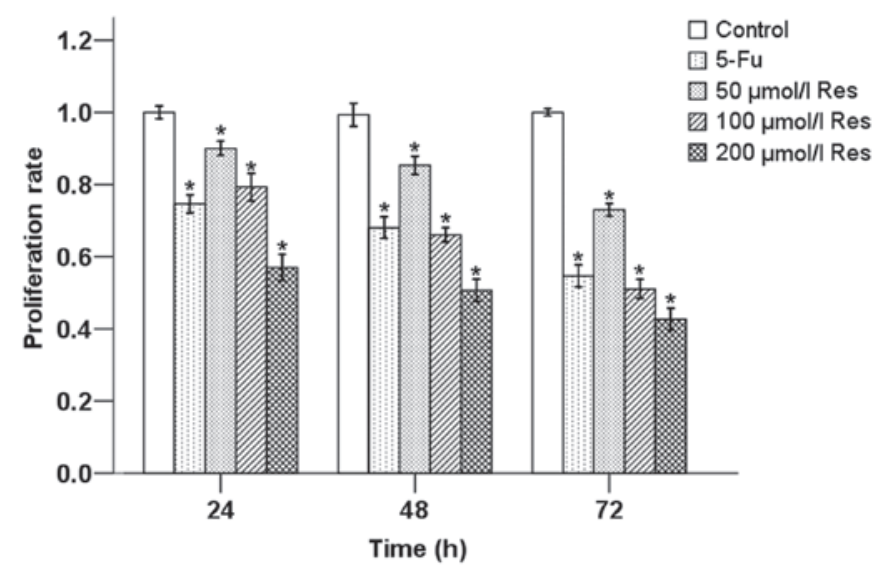

Figure 2. Resveratrol (Res) reduces the proliferative ability of pancreatic cancer (PC) cells. The percentage of growth inhibition of human PC MIA $\mathrm{PaCa}-2$ cells treated with increasing concentrations of Res $(0-200 \mu \mathrm{mol} / \mathrm{l})$ at 24, 48 and 72 h. ${ }^{*} \mathrm{P}<0.05$ as compared to the negative control group. 5-FU, 5-fluorouracil.

\section{Results}

Effect of 5-Fu on the proliferation of MIA PaCa-2PC cells. The cytotoxicity of 5-Fu was first determined using the MTT assay. MIA PaCa- 2 cells were treated with various concentrations $(0,0.25,0.5,0.75$ and $1.0 \mathrm{mg} / \mathrm{ml})$ of 5 -Fu for 24,48 and $72 \mathrm{~h}$. The results demonstrated that the proliferative ability of MIA PaCa- 2 cells is decreased in response to $5-\mathrm{Fu}$ treatment in a time- and dose-dependent manner. The half maximal inhibitory concentration $\left(\mathrm{IC}_{50}\right.$ ) for MIA PaCa-2 cells was $\sim 0.75 \mathrm{mg} / \mathrm{ml}$ of 5 -Fu (Fig. 1), and this concentration exhibited no additional cytotoxic effects on the MIA PaCa-2 cells (data not shown). Therefore, $0.75 \mathrm{mg} / \mathrm{ml} \mathrm{5-Fu} \mathrm{was} \mathrm{used} \mathrm{for} \mathrm{the}$ subsequent experiments.

Effect of Res on the proliferation of pancreatic cancer cells. We next treated MIA PaCa-2 cells with different concentrations of Res (50, 100 and $200 \mu \mathrm{mol} / \mathrm{l})$. As shown in Fig. 2, cell

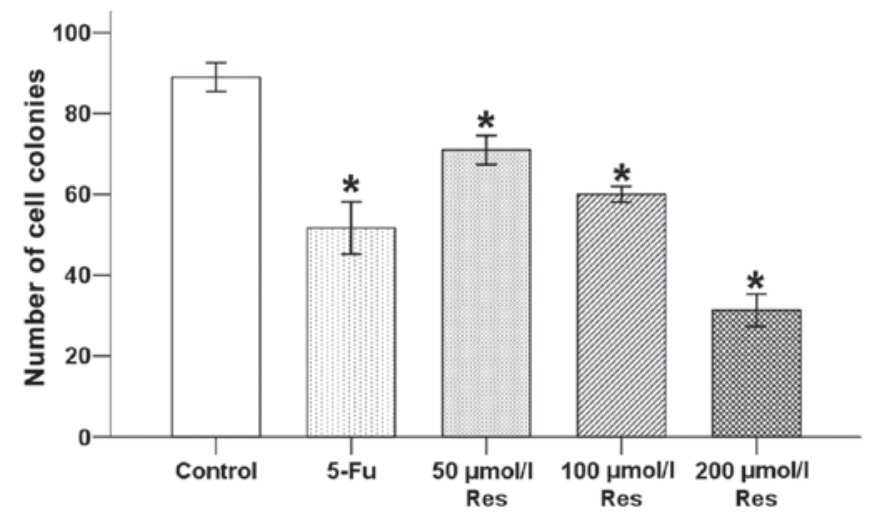

Figure 3. The effects of different treatments on the colony formation ability of MIA PaCa-2 cells. 5-Fluorouracil (5-Fu) and resveratrol (Res) reduce the number of cell colonies. ${ }^{*} \mathrm{P}<0.05$ as compared to the negative control group.

proliferation was inhibited by the administration of Res in a dose- and time-dependent manners.

In addition, the number of cell colonies in the three Res treatment groups and the 5-Fu group was significantly decreased as compared to the negative control group (Fig. 3). The colonies in the high concentration Res group were fewer than those in the other Res groups. The colony number was gradually reduced with the increasing Res concentration, indicating that Res inhibits the proliferation of PC cells in a concentration-dependent manner.

Effects of Res and 5-Fu on apoptosis of cancer cells. Cell apoptosis was assessed by the Annexin V-FITC and PI double staining method. The results are shown in Fig. 4. The apoptotic rate was significantly increased in cells treated with $5-\mathrm{Fu}$ and Res, and the number of apoptotic cells increased with the increasing concentrations of Res. This result suggested that Res plays an important role in promoting apoptosis in MIA $\mathrm{PaCa}-2 \mathrm{PC}$ cells, and in a concentration-dependent manner.

Effects of Res on the protein and mRNA expression of Ihh, Ptch and Smo. After cells were treated with Res and 5-Fu for $24 \mathrm{~h}$, the protein and mRNA levels of Ihh, Ptch and Smo were measured by western blot analysis (Fig. 5A) and RT-qPCR (Fig. 5B). Compared to the negative control group, the expression of Ihh, Ptch and Smo was decreased in all Res groups and the 5-Fu group, at both the protein and mRNA levels. The effects of Res were dose-dependent.

\section{Discussion}

The pathogenesis of pancreatic cancer remains unknown. Its progresses rapidly with a poor prognosis and a high mortality rate. It is often diagnosed at a late stage and the outcome of surgery is commonly unsatisfactory. Currently, the standard treatments for PC are radiotherapy and chemotherapy, and there are no effective drugs for this disease (3). A new comprehensive and effective therapy is thus urgently needed.

Res, or trans-3,5,4-trihydroxy-stilbene $\left(\mathrm{C}_{14} \mathrm{H}_{12} \mathrm{O}_{3}\right)$ has a relative molecular mass of 228.25, and contains non-flavonoid polyphenol compounds in its stilbene structure. It has been reported that Res plays an antagonistic role in numerous 

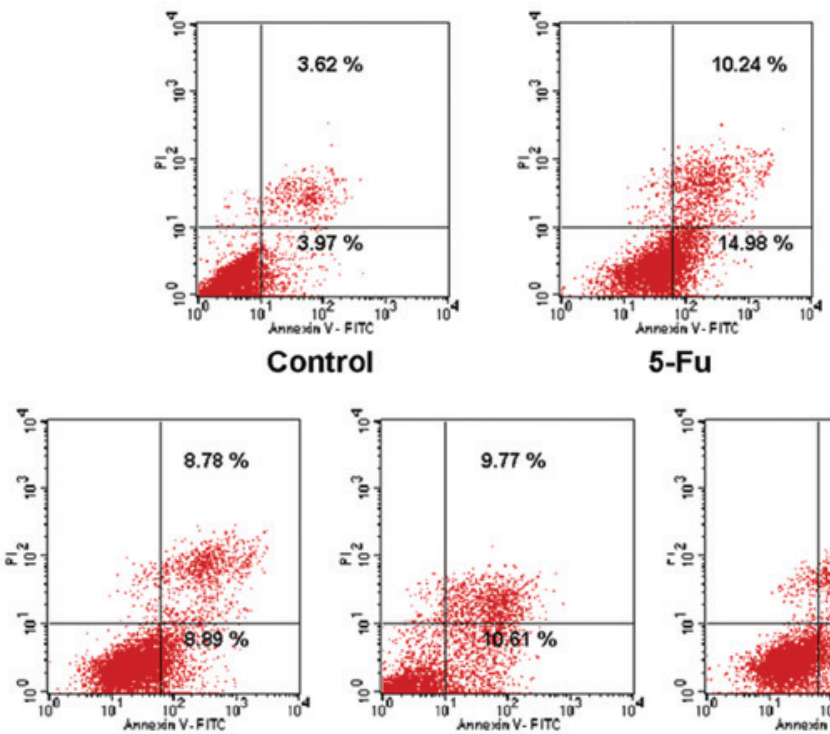

$50 \mu \mathrm{mol} / \mathrm{l}$ Res

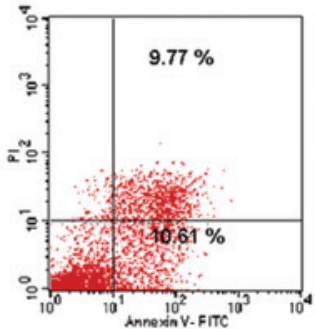

$100 \mu \mathrm{mol} / \mathrm{l}$ Res

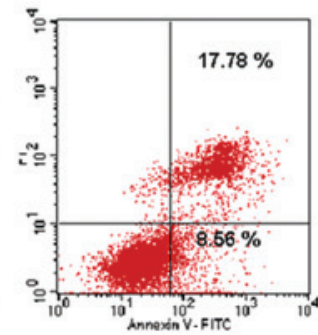

$200 \mu \mathrm{mol} / \mathrm{l}$ Res

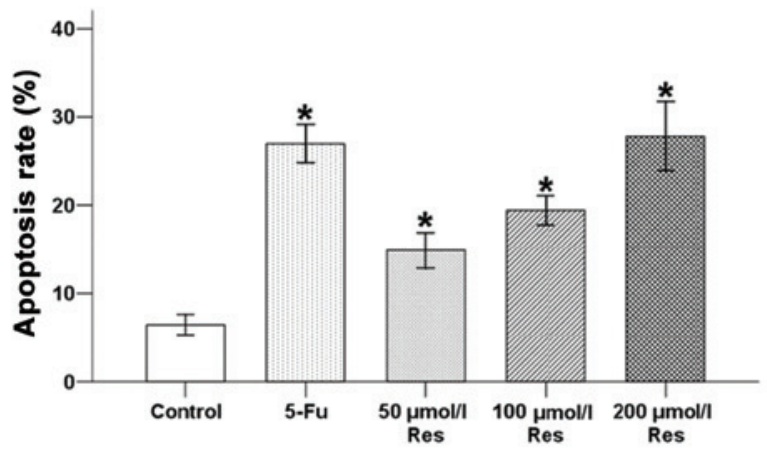

Figure 4. 5-Fluorouracil (5-Fu) and resveratrol (Res) increase the apoptotic rate of MIA PaCa-2 pancreatic cancer cells. The cells were treated with 5-Fu and increasing concentrations of Res $(0,50,100$ and $200 \mu \mathrm{M})$ for $24 \mathrm{~h}$. ${ }^{*} \mathrm{P}<0.05$ as compared to the negative control group.

A

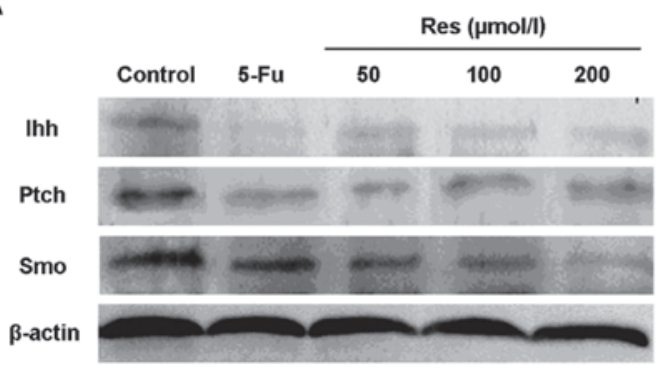

B

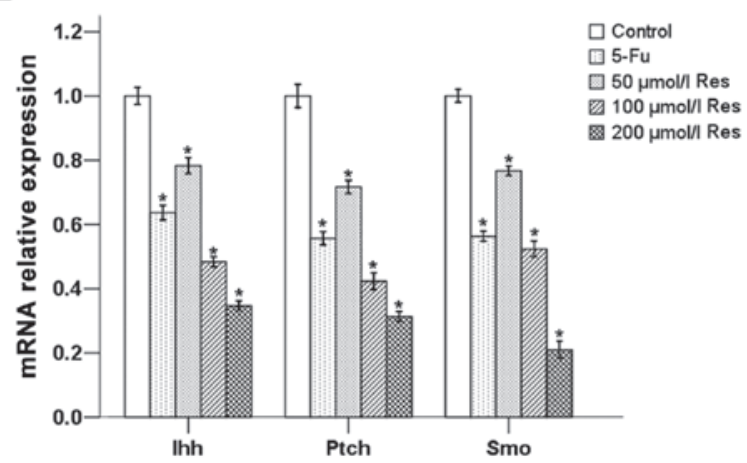

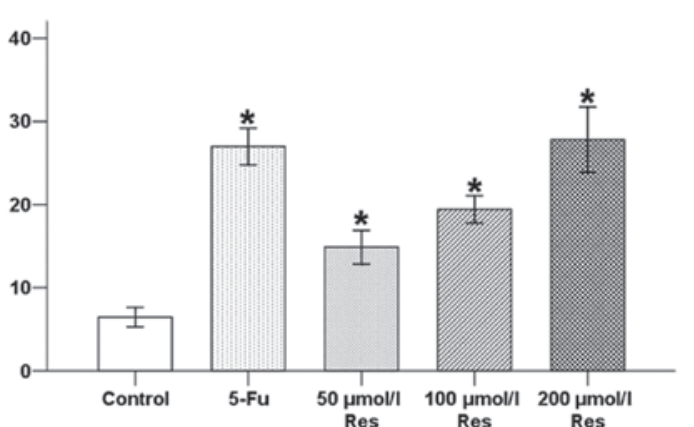

Res Res 
PaCa- 2 cell line with Res at three different concentrations, cell proliferation was measured by the MTT assay, and cell apoptosis was detected by flow cytometry. The results showed that Res can inhibit cell proliferation in a time- and dosedependent manner, and that the inhibitory effects of different concentrations of Res are significantly different. In addition, Res significantly promoted cell apoptosis. The rate of apoptosis increased with the resveratrol concentration. In general, the results of our experiments confirm that Res inhibits the growth of MIA PaCa-2 PC cells in vitro, by decreasing cell proliferation rates and promoting cell apoptosis.

The imbalance between cell proliferation and apoptosis is often due to a genetic defect in cellular control mechanisms. This imbalance may be induced by the mutation of specific genes, and mutations in signal transduction genes are of key importance in this context. The Hh signal transduction pathway is closely associated with the occurrence and development of $\mathrm{PC}$. The Hh signaling pathway, which is quiescent in the adult healthy pancreas, was shown to be active in pancreatic cancer and to relate to cancer progression (18). Abnormal expression of the $\mathrm{Hh}$ ligand or the downstream signaling pathway proteins, as well as dysfunctions in these pathway components, can induce tumorigenesis. The potential mechanisms include mutations in the Smo gene, loss of function of Ptch, etc. Yang et al (5) identified 54 cases of PC using immunohistochemical methods, and high expression of the proteins Ihh, Ptch and Smo was detected in these cases. In the corresponding adjacent tissues, the proteins were found expressed only in the islet cells; no expression was observed in healthy pancreatic ductal tissues. Our experiments show that the mRNA and protein expression of Ptch, Smo and Ihh are significantly reduced upon Res treatment. Their expression levels gradually decrease as the Res concentration increases, indicating that Res influences the Ptch, Smo and Ihh expression in a concentration-dependent manner. Res may thus block the Hh signaling pathway in PC cells by downregulating the expression of Ihh, Ptch and Smo.

In conclusion, Res inhibits the growth of human MIA $\mathrm{PaCa}-2$ cells in vitro by inhibiting cell proliferation and promoting cell apoptosis. This effect may related to an inhibition of the Hh signaling pathway through downregulation of Ihh, Ptch and Smo at the mRNA and protein levels. Res treatment may thus be a novel option for therapy of PC via the inhibition of the Hh signaling pathway.

\section{Acknowledgements}

We greatly appreciate the technical assistance offered by staff members of the Biology and Genetics Laboratory, at the Xi'an Jiaotong University. This study was supported by grants from the National Natural Science Foundation of China (nos., 81172360 and 81301846).

\section{References}

1. Siegel R, Naishadham D and Jemal A: Cancer statistics, 2013. CA Cancer J Clin 63: 11-30, 2013.

2. Mancuso A, Calabro F and Sternberg CN: Current therapies and advances in the treatment of pancreatic cancer. Crit Rev Oncol Hematol 58: 231-241, 2006.

3. Krug S and Michl P: New developments in pancreatic cancer treatment. Minerva Gastroenterol Dietol 58: 427-443, 2012.

4. Li X, Ma Q, Xu Q, Liu H, Lei J, Duan W, Bhat K, Wang F, Wu E and Wang Z: SDF-1/CXCR4 signaling induces pancreatic cancer cell invasion and epithelial-mesenchymal transition in vitro through non-canonical activation of Hedgehog pathway. Cancer Lett 322: 169-176, 2012.

5. Yang Y, Tian X, Xie X, Zhuang Y, Wu W and Wang W: Expression and regulation of hedgehog signaling pathway in pancreatic cancer. Langenbecks Arch Surg 395: 515-525, 2010.

6. Dosch JS, Pasca di Magliano M and Simeone DM: Pancreatic cancer and hedgehog pathway signaling: new insights. Pancreatology 10: 151-157, 2010.

7. Villavicencio EH, Walterhouse DO and Iannaccone PM: The sonic hedgehog-patched-gli pathway in human development and disease. Am J Hum Genet 67: 1047-1054,2000.

8. Giovinazzo G, Ingrosso I, Paradiso A, De Gara L and Santino A: Resveratrol biosynthesis: plant metabolic engineering for nutritional improvement of food. Plant Foods Hum Nutr 67: 191-199, 2012.

9. Fulda S: Resveratrol and derivatives for the prevention and treatment of cancer. Drug Discov Today 15: 757-765, 2010.

10. Jha RK, Ma Q, Sha H and Palikhe M: Emerging role of resveratrol in the treatment of severe acute pancreatitis. Front Biosci (Schol Ed) 2: 168-175, 2010.

11. Pervaiz S and Holme AL: Resveratrol: its biologic targets and functional activity. Antioxid Redox Signal 11: 2851-2897, 2009.

12. Li W, Ma J, Ma Q, Li B, Han L, Liu J, Xu Q, Duan W, Yu S, Wang $\mathrm{F}$ and $\mathrm{Wu} \mathrm{E}$ : Resveratrol inhibits the epithelial-mesenchymal transition of pancreatic cancer cells via suppression of the PI-3K/Akt/NF-kB pathway. Curr Med Chem 20: 4185-4194, 2013.

13. Livak KJ1 and Schmittgen TD: Analysis of relative gene expression data using real-time quantitative PCR and the 2(-Delta Delta C(T)) method. Methods 25: 402-408. 2001.

14. Singh CK, George J and Ahmad N: Resveratrol-based combinatorial strategies for cancer management. Ann N Y Acad Sci 1290: 113-121, 2013

15. Aluyen JK, Ton QN, Tran T, Yang AE, Gottlieb HB and Bellanger RA: Resveratrol: potential as anticancer agent. J Diet Suppl 9: 45-56, 2012.

16. Yang Q, Wang B, Zang W, Wang X, Liu Z, Li W and Jia J: Resveratrol inhibits the growth of gastric cancer by inducing G1 phase arrest and senescence in a sirt1-dependent manner. PLoS One 8: e70627, 2013.

17. Ji Q, Liu X, Fu X, Zhang L, Sui H, Zhou L, Sun J, Cai J, Qin J, Ren J and Li Q: Resveratrol inhibits invasion and metastasis of colorectal cancer cells via MALAT1 mediated Wnt/ $\beta$-catenin signal pathway. PLoS One 8: e78700, 2013.

18. Olive KP, Jacobetz MA, Davidson CJ, Gopinathan A, McIntyre D, Honess D, Madhu B, Goldgraben MA, Caldwell ME, Allard D, Frese KK, Denicola G, Feig C, Combs C, Winter SP, Ireland-Zecchini H, Reichelt S, Howat WJ, Chang A, Dhara M, Wang L, Ruckert F, Grutzmann R, Pilarsky C, Izeradjene K, Hingorani SR, Huang P, Davies SE, Plunkett W, Egorin M, Hruban RH, Whitebread N, McGovern K, Adams J, Iacobuzio-Donahue C, Griffiths J and Tuveson DA: Inhibition of Hedgehog signaling enhances delivery of chemotherapy in a mouse model of pancreatic cancer. Science 324: 1457-14611, 2009. 